\title{
Perifoveal vascular leakage and macular oedema after intracapsular cataract extraction
}

\author{
TRAVIS A. MEREDITH, KENNETH R. KENYON, LAWRENCE J. SINGERMAN, \\ AND STUART L. FINE \\ From the Wilmer Ophthalmological Institute, Baltimore, Maryland
}

Cystoid macular oedema is now a commonly recognized complication after cataract extraction. Irvine (1953) described a syndrome of spontaneous rupture of the anterior hyaloid face with vitreous adhesions to the wound, irritability of the eye, and reduction of vision secondary to macular changes. Gass and Norton (1966) correlated the typical ophthalmoscopic appearance of dilated spaces in the central macula with late pooling of fluorescein dye in these spaces after intravenous injection. Gass and Norton (1969) described the clinical course of the syndrome in a referral population of 48 patients. Typically, visual acuity was initially good and then declined 4 to 12 weeks after cataract extraction. Forty per cent of those eyes had the vitreous attached to the fosterior surface of the wound and many eyes had inflammatory cells in the posterior vitreous. Other authors have reported that this syndrome occurs in approximately 5 to 7 per cent of patients after cataract extraction (Gehring, 1968; Maumenee and Meredith, 1974).

Irvine, Bresky, Crowder, Forster, Hunter, and Kulvin (197I) reported on 100 aphakic patients who had angiograms 4 to 16 weeks after cataract extraction. Forty per cent of the angiograms showed dye leakage in the macula with a maximum incidence six to eight weeks postoperatively. When repeat angiograms were available at the twentieth week postoperatively, two-thirds of the patients initially demonstrating leakage had reverted to normal. Two subsequent studies (Hitchings, Chisholm, and Bird, 1975; Hitchings and Chisholm, 1975) demonstrated an approximately 50 per cent incidence of fluorescein leakage from perifoveal capillaries six weeks postoperatively.

The present prospective study was undertaken to investigate further the incidence and time course of perifoveal capillary leakage atter cataract surgery. Factors associated with the presence of leakage were also investigated.

Presented in part at the 34th annual meeting of the Wilmer Residents Association, 18 April 1975

Address for reprints: Travis A. Meredith, MD, Department of Ophthalmology, Medical College of Wisconsin, 8700 West Wisconsin Avenue, Milwaukee, Wisconsin 53226, USA

\section{Patients and methods}

During the I5-month period from December 1973 to February 1975, fluorescein angiograms were performed on patients two weeks after cataract extraction and, if possible, again six weeks postoperatively. Intracapsular extractions were attempted in all cases. Approximately 50 per cent of the patients undergoing cataract extraction during this period had angiography. Reasons for patients not being included in the study were their inability to co-operate, refusal to participate, or inability to schedule angiography. The patients comprising this sample did not differ in age, race, or sex from the total operated population during that period, so that no bias of ascertainment existed. Furthermore, the percentage of patients in this sample who had operative complications was the same as a group of unselected patients undergoing cataract extraction (Maumenee and Meredith, 1974).

Before surgery all patients studied had a complete medical history; physical examinations included blood count and urine analysis. Each had a complete ocular history and examination which included visual acuity, refraction, external examination, slit-lamp examination, gonioscopy, central and peripheral visual fields, and dilated fundus examination. Thorough ocular examinations were carried out at the time of discharge and at each postoperative visit when angiography was performed.

Angiography was performed by the standard technique used at the Wilmer Ophthalmological Institute; $5 \mathrm{ml}$ of 10 per cent sodium fluorescein is injected intravenously, photographs of the posterior pole are taken 5 seconds after injection and every second thereafter for 15 more seconds. Additional photographs are taken up to one minute at 3 to 5 second intervals, and repeated at 2, 5, 10, and 30 minutes. The Zeiss fundus camera with Allen stereo separator and Kodak Tri-X film were used. Stereo colour fundus photographs were taken in all cases. Angiograms were evaluated independently by two separate observers who had no knowledge of the patient's systemic condition or ocular history.

Patients were not included in the study if there was any ocular condition present known to be associated with macular oedema. Thus, three patients with mild diabetic retinopathy, one patient with an old branch vein occlusion, and one patient with retinitis pigmentosa were excluded although they all demonstrated leakage of fluorescein dye from perifoveal capillaries Patients 
were also excluded if they had macular scarring from other lesions which interfered with the interpretation of the angiogram. Several angiograms were excluded because their quality was inadequate for precise interpretation.

\section{Results}

The 44 patients included in this study comprised 20 men and 24 women; 33 patients were Caucasian, and I I were Black. The average age was 63 years (range 42 to 79 years).

Fluorescein angiograms done two weeks after cataract extraction were available on 50 eyes of the 44 patients. At this time, perifoveal capillary leakage was present in 30 out of 50 eyes ( 60 per cent) and in 25 of 44 patients ( 56 per cent). Six weeks postoperatively, follow-up angiograms were available on 17 eyes. Twelve of these eyes had initially demonstrated leakage at two weeks and I I continued to leak at six weeks. None of the five patients negative at six weeks had shown leakage in angiograms two weeks after surgery.

The pattern of leakage varied widely throughout the series. Some patients showed only mild or moderate perifoveal capillary staining (Fig. I) three to five minutes after injection, whereas others demonstrated extensive leakage with marked pooling in cystoid spaces in films taken 15 to 20 minutes postinjection. None of the patients with leakage demonstrated the dilatation of perifoveal vasculature noted in some cases of chronic cystoid oedema. Thus, perifoveal vascular dilatation may indicate a degree of chronicity that may be the hallmark of more severely damaged vessels.

\section{VASCULAR DISEASE}

Fourteen patients had either demonstrable cardiovascular disease or diabetes mellitus. Seven of these patients had adult-onset diabetes mellitus requiring medication but did not have background diabetic retinopathy. Three had hypertension requiring medication. Two had both diabetes and hypertension. Two had arteriosclerosis manifested by cardiac changes. Thirteen of the 14 patients with vascular disease (93 per cent) demonstrated perifoveal capillary leakage. Of the 30 patients without cardiovascular disease, only 12 (40 per cent) showed leakage. This difference is statistically significant at the $\mathrm{P}<0.001$ level.

\section{AGE}

Of I I patients under the age of 60 years, only two (i 8 per cent) demonstrated dye leakage, and both had cardiovascular disease. Of the 33 patients who were 60 years of age or older, 23 (70 per cent) demonstrated leakage (Fig. 2a). When the data are corrected by excluding patients with the previously identified risk factor of vascular dis zase which is associated with leakage, none of the nine patients under 6o years showed abnormal capillary permeability, while 12 of the 2 I patients ( 57 per cent) who were 60 years of age or older demonstrated leakage (Fig. 2b). This difference is statistically significant at the $\mathrm{P}<0.00 \mathrm{I}$ level.

\section{BILATERALITY}

Six patients had bilateral cataract extractions. Five patients were positive for abnormal leakage in both

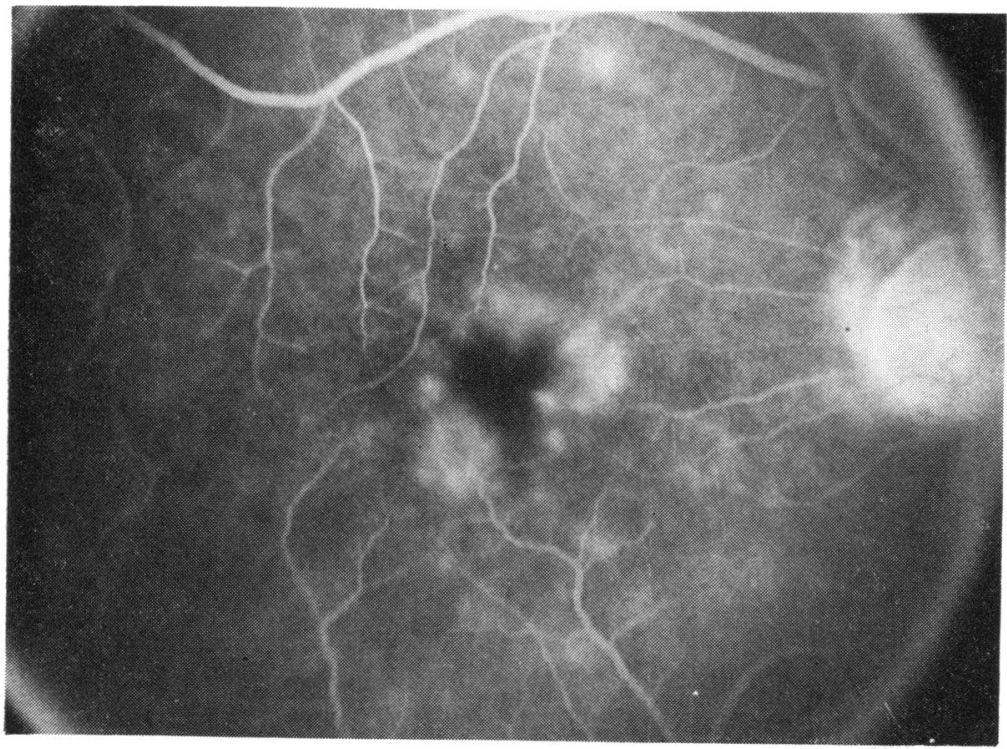

FIG. I Fluorescein angiography at two weeks after uncomplicated intracapsular cataract extraction. Moderate perifoveal leakage is apparent seven minutes after fluorescein injection 


\begin{abstract}
FIG. 2a Age distribution of all patients with leakage (solid bar) and without leakage (hollow bar) at two weeks postoperatively

FIG. 2b Age distribution of patients not having systemic vascular disease with leakage (solid bar) and without leakage (hollow bar) at two weeks postoperatively
\end{abstract}
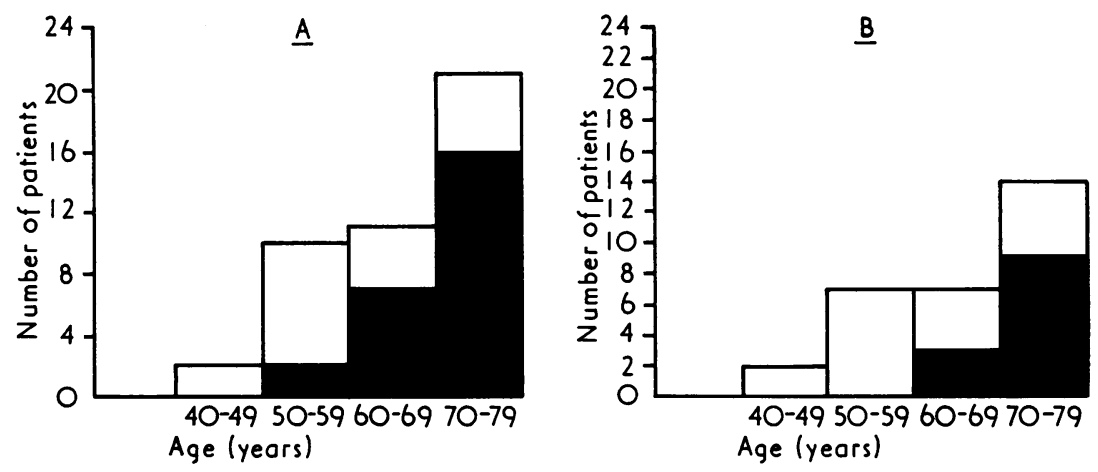

eyes, and three of these five had vascular disease. One patient did not show leakage in either eye.

\section{ANTERIOR VITREOUS FACE}

Specific comments about the condition of the anterior hyaloid face at the time of initial angiography were available for 34 eyes. Of 30 eyes with an intact vitreous face, I6 (53 per cent) showed leakage. Of four eyes with ruptured anterior hyaloid membrane, three of four ( 75 per cent) demonstrated leakage.

Late rupture of the anterior hyaloid face occurred in three patients with resultant vitreous to the wound. All three patients had previously developed vision equal to or better than $20 / 40$, and all had demonstrated perifoveal capillary leakage before the time of the vitreous face rupture. Two of the three patients suffered a later decline in visual acuity associated with clinically apparent cystoid macular oedema. A fourth patient who had initially demonstrated no dye leakage developed a ruptured anterior face resulting in complete filling of the anterior chamber with vitreous. No change in vision resulted, and the follow-up angiogram remained negative.

\section{OPERATIVE AND POSTOPERATIVE COMPLICATIONS}

Two patients had inadvertent extracapsular extractions with vitreous loss. Both had anterior vitrectomies, and neither had vitreous to the wound. One had abnormal capillary leakage at the two-week angiogram, and one had not. Two patients had inadvertent extracapsular extractions without loss of vitreous, and neither had perifoveal leakage. Two patients developed postoperative hyphaemas, and both demonstrated leakage on angiography. One patient was discovered to have a cyclodialysis cleft postoperatively. The intraocular pressure was normal on the day of an angiogram which showed no leakage.

\section{VISUAL ACUITY}

At the time of initial angiography, there was no difference in the visual acuities between the group of patients demonstrating leakage and those who did not (Fig. 3a). At six weeks, all five eyes not obtaining acuity of $20 / 40$ or better had shown leakage in the two-week angiogram (Fig. $3 b$ ).

\section{ANTERIOR SEGMENT}

Subjective assessment of external injection and anterior chamber reaction (rated $\circ$ to $4+$ ) were made at the time of discharge from the hospital and at each postoperative visit. Most patients demonstrated 0 to $\mathbf{I}+$ inflammation at the time of initial angiography, and there was no difference in apparent inflammation between the group of patients who showed dye leakage and the group who did not. Of the II patients who demonstrated leakage at the six-week angiogram, only one was judged to have either external or anterior chamber inflammation.

\section{INTRAOCULAR PRESSURE}

Intraocular pressure was determined preoperatively, at hospital discharge, and at each postoperative visit. The difference in the preoperative and postoperative values was determined for each patient. The range of values and the mean values were the same for the group of patients who showed leakage as for the group who did not, both at discharge and at time of angiography. There were no cases of persistent hypotony in either group.

\section{Discussion}

This study confirms previous reports showing a high incidence of fluorescein leakage from perifoveal capillaries after cataract extraction (Irvine and others, I97 I ; Hitchings and others, I 975 ; Hitchings and Chisholm, I975). These earlier reports have 

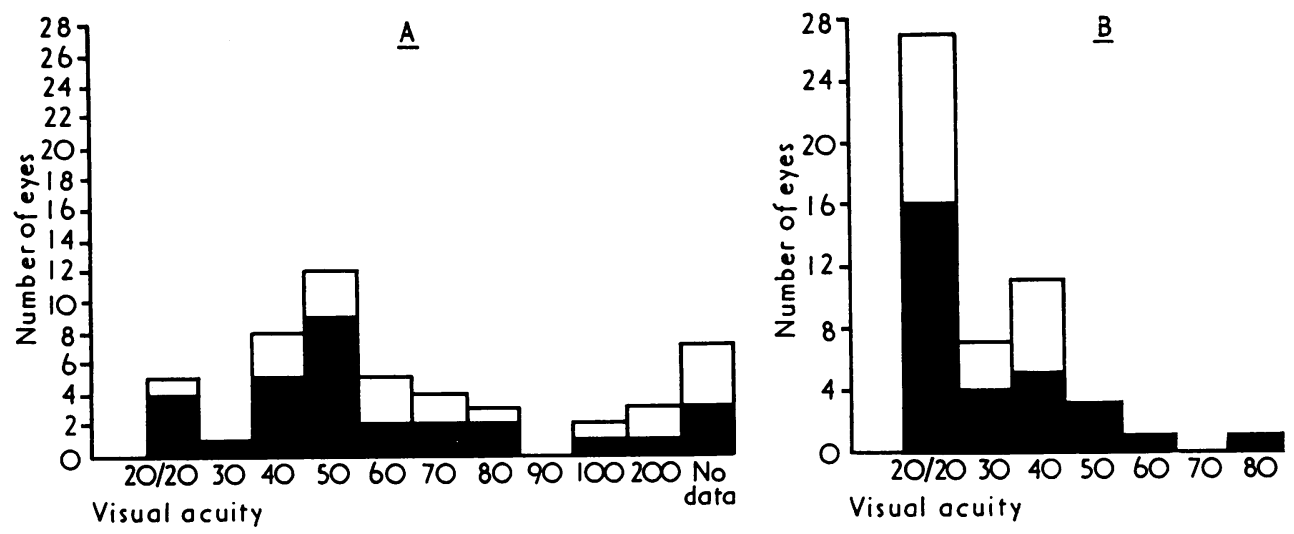

FIG. 3a Distribution of visual acuities two weeks postoperatively in all eyes with leakage (solid bar) and without leakage (hollow bar)

FIG. 3b Distribution of visual acuities six weeks postoperatively in all eyes with leakage (solid bar) and without leakage (hollow bar)

either indicated or assumed that leakage would reach maximal incidence at six weeks postoperatively, a time when patients are frequently found to have symptomatic reduction in visual acuity with ophthalmoscopically visible cystoid spaces in the macula. Our study indicates that leakage consistently develops within two weeks after operation and almost uniformly continues to be present six weeks postoperatively (I I/ I 2 eyes). In particular, we did not encounter leakage at six weeks that had not been present at two weeks, and there is no statistical difference in the percentage of patients found to be leaking at two weeks in our study and at six weeks in the studies by Hitchings and others (1975) and Hitchings and Chisholm (1975). We have subsequently observed fluorescein leakage to be present as early as five days after cataract extraction (unpublished observation).

A study (Klein and Yannuzzi, 1976) which noted only classic 'cystoid oedema of the macula' found a small percentage ( 5 per cent) of positive angiograms in the first week. Most of our cases demonstrated only mild degrees of perifoveal leakage in the early angiograms. Some of these progressed to demonstrate the more advanced changes of dilated cystic spaces in the macula on the six-week angiograms, while in others the amount of leakage remained the same or decreased.

Vascular disease in the form of hypertension, arteriosclerosis, and diabetes mellitus was shown to be a significant factor associated with altered capillary permeability in the early postoperative period. This implies that pre-existing disease of small vessels renders them more susceptible to develop leakage after cataract extraction, although leakage from these vessels before lens extraction cannot be ruled out.

The firm association of leakage with the factors of vascular disease and age, coupled with the negative correlations with operative and postoperative complications, inflammation, and intraocular pressure suggest that constitutional factors are important in precipitating abnormal vascular permeability during the early postoperative period. The identical angiographic behaviour of both eyes in each of the six patients studied bilaterally further supports this conclusion.

Perifoveal capillary leakage is compatible with $20 / 20$ acuity. However, all five patients in this series who were not correctible to $20 / 40$ six weeks postoperatively had had leakage demonstrable by the second postoperative week. If the criteria of reduction of vision and fluorescein dye leakage from perifoveal capillaries after cataract extraction are used to define the Irvine-Gass syndrome, then the present series demonstrates an approximately Io per cent incidence.

While our data indicate that age and vascular disease are important factors in altered permeability of perifoveal capillaries in the early postoperative period, we are unable to identify precisely the cause of persistent dye leakage and decreased visual acuity. Gass and Norton (1969) identified operative complications, irido-vitreal abnormalities, inflammation, and vascular disease in a large proportion of their patients with aphakic macular oedema. Our patients with operative and postoperative complications did not consistently demonstrate early dye leakage, but the number of such patients in this series is too small to draw statistically significant conclusions. 
The precise role of inflammation is also not clear. Our patients with dye leakage did not show any more anterior segment inflammation than did patients without leakage. Ten of II patients with leakage apparent in the six-week angiogram had no detectable inflammation at that time. Other reports have also indicated that clinical signs of anterior segment inflammation are often absent (Gass and Norton, 1969; Tolentino and Schepens, 1965). Vitreous inflammation, particularly the presence of cells in the posterior vitreous, has been considered important (Gass and Norton, 1969). In histopathologically studied cases, inflammatory changes of cyclitis, vitritis, and retinal phlebitis have been noted (Michels, Green, and Maumenee, 1971; Norton, Brown, Carlson, Pilger, and Riffenburgh, 1975; Martin, Green, and Martin, personal communication). These findings of widespread intraocular inflammation seemingly complement the clinical indications of the importance of vascular factors in the development of this syndrome.

Once leakage is established, it is possible that its persistence in a degree sufficient to cause decreased visual acuity is promoted by inflammation and/or irido-vitreal abnormalities. This possibility is illustrated in one of our patients, a 78-year-old diabetic man who had 20/40 vision despite perifoveal capillary leakage six weeks postoperatively. Five months postoperatively, however, delayed rupture of the anterior hyaloid face had occurred with vitreous to the wound, irritation, and subsequent reduction of acuity to 20/70. Angiography again demonstrated perifoveal capillary leakage.

Vascular factors may be important in sustaining leakage as well as causing it early in the postoperative period. Gass and Norton (1969) demonstrated vascular disease in 75 per cent of their patients with cystoid macular oedema and in 13 of 14 patients whose oedema did not resolve within the period of their study. It is possible that vascular factors also account for sufficient leakage to cause decreased acuity in quiet eyes with no inflammation or irido-vitreal abnormalities. Aphakic cystoid macular oedema may thus represent the final common pathway of several separate although sometimes related factors which result in altered perifoveal capillary permeability.

\section{Summary}

Perifoveal capillary leakage of fluorescein was demonstrated in 60 per cent of 50 eyes when angiography was performed two weeks after cataract extraction. Repeat angiography six weeks postoperatively in 17 eyes demonstrated persistence of already established leakage in 11 of 12 eyes and no new leakage in five eyes previously negative. Cystoid macular oedema with visual acuity of less than 20/40 six weeks postoperatively occurred in five eyes (10 per cent).

Eyes of patients with vascular disease and those patients of 60 years or older were found to have altered vascular permeability significantly more frequently. Inflammation was no more severe or prevalent in those patients who demonstrated leakage and no inflammation was clinically apparent in IO of II eyes demonstrating dye leakage six weeks postoperatively. We conclude that the constitutional factors of age and vascular disease are of prime importance in causing altered vascular permeability in the early postoperative period after cataract extraction; factors causing sustained leakage with reduction of visual acuity were not demonstrated.

We thank Dr Arnall Patz and Dr Thomas Aaberg for reviewing the manuscript, Mrs Dolores Rytel, Mrs Judy Belt, and Mr Terry George, RBP, for photographic and angiographic studies, and Miss Peggy Rasnake and Miss Cathy Vecchio for preparation of the manuscript.

\section{References}

GASS, J. D. M., and NORTON, E. W. D. (1966) Arch. Ophthal. (Chic.), 76, 647

, and - (1969) Trans. Amer. Acad. Ophthal. Otolaryng., 73, 665

GeHRING, J. R. (1968) Arch. Ophthal. (Chic.), 80, 626

HitChingS, R. A., and CHISHOLM, I. H. (1975) Brit. F. Ophthal., 59, 444

- - and BIRD, A. c. (1975) Invest. Ophthal., 14, 68

IRVINE, S. R. (1953) Amer. F. Ophthal., 36, 599

, BRESKY, R., CROWDER, B. M., FORSTER, R. K., HUNTER, D. M., and KULVIN, S. M. (1971) Ann. Ophthal., 3, I234

KLEIN, R. M., and YANNUZZI, L. (1976) Amer. F. Ophthal., 81, 614

MARTIN, N. F., GREEN, W. R., and MARTIN, L. W. (Personal communication)

MAUMENEE, A. E., and Meredith, T. A. (1974) 'Current Concepts in Cataract Surgery', eds J. M. Emery and

D. Paton, p. 423. Mosby, St Louis

MICHELS, R. G., GREEN, W. R., and MAUMENEE, A. E. (1971) Ophthal. Surg., 2, 217

NORTON, A. L., BROWN, w. J., CARLSON, M., PILGER, I. S., and RIFFENBURGH, R. S. (1975) Amer. F. Ophthal., 80, 96

TOLENTINo, F. I., and SChePENS, C. L. (1965) Arch. Ophthal., 74, 718 